\title{
ON UNIFORM DISTRIBUTION MODULO A SUBDIVISION
}

\author{
W. J. LEVEQUE
}

1. Let $\Delta$ be a subdivision of the interval $(0, \infty): \Delta=\left(z_{0}, z_{1}, \cdots\right)$, where

$$
0=z_{0}<z_{1}<\cdots \text { and } \lim _{n \rightarrow \infty} z_{n}=\infty \text {. }
$$

For $z_{n-1} \leq x<z_{n}$, put

$$
[x]_{\Delta}=z_{n-1}, \quad \delta(x)=z_{n}-z_{n-1},\langle x\rangle_{\Delta}=\frac{x-[x]_{\Delta}}{\delta(x)}, \phi(x)=n+\langle x\rangle_{\Delta},
$$

so that $0 \leq\langle x\rangle_{\Delta}<1$. Let $\left\{x_{k}\right\}$ be an increasing sequence of positive numbers. If the sequence $\left\{\left\langle x_{k}\right\rangle_{\Delta}\right\}$ is uniformly distributed over $[0,1]$, in the sense that the proportion of the numbers $\left\langle x_{1}\right\rangle_{\Delta}, \cdots,\left\langle x_{k}\right\rangle_{\Delta}$ which lie in $[0, \alpha)$ approaches $\alpha$ as $k \rightarrow \infty$, for each $\alpha \in[0,1)$, then we shall say that the sequence $\left\{x_{k}\right\}$ is uniformly distributed modulo $\Delta$. If $\Delta$ is the subdivision $\Delta_{0}$ for which $z_{n}=n$, this reduces to the ordinary concept of uniform distribution $(\bmod 1)$, since then $[x]_{\Delta}=$ $[x], \delta(x)=1$ for all $x$, and $\langle x\rangle_{\Delta}=x-[x]$ is the fractional part of $x$. Even in other cases, the generalization is more apparent than real, since the uniform distribution of one sequence $(\bmod \Delta)$ is equivalent to the uniform distribution of another sequence $(\bmod 1)$. But most of the known theorems concerning uniform distribution $(\bmod 1)$ are not applicable to the sequences $\left\{\left\langle x_{k}\right\rangle_{\Delta}\right\}$, if $\Delta$ is not $\Delta_{0}$, for in such theorems $x_{k}$ is ordinarily taken to be the value $f(k)$ of a function whose derivative exists and is monotonic for positive $x$. Here, on the other hand, $\left\langle x_{k}\right\rangle_{\Delta} \equiv \phi\left(x_{k}\right)(\bmod 1)$, and $\phi$, although a continuous polygonal function, is not necessarily everywhere differentiable; and unless $\delta(x)$ is assumed monotonic, $\phi^{\prime}$ is not monotonic even over the set on which it exists. This lack of monotonicity introduces serious difficulties; it is the object of the present work to show how they can be dealt with in certain cases.

For brevity, "uniformly distributed" will be abbreviated to "u.d.". The sym-

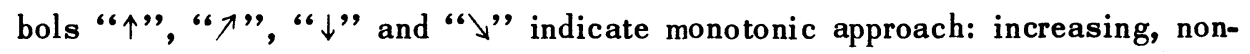
decreasing, decreasing, and non-increasing, respectively.

Received December 3, 1952.

Pacific J. Math. 3 (1953), 757-771 


\section{Put}

$$
N(\alpha, x)=\sum_{\substack{x_{k} \leq x \\\left\langle x_{k}\right\rangle\langle<}} 1, \quad N(x)=N(1, x) ;
$$

then $\left\{x_{k}\right\}$ is u.d. $(\bmod \Delta)$ if and only if, for each $\alpha \in[0,1)$,

$$
\lim _{x \rightarrow \infty} \frac{N(\alpha, x)}{N(x)}=\alpha \text {. }
$$

THE OREM 1. A necessary condition that $\left\{x_{k}\right\}$ be u.d. $(\bmod \Delta)$ is that

$$
N\left(z_{n+1}\right) \sim N\left(z_{n}\right)
$$

as $n \longrightarrow \infty$.

For suppose that $\left\{x_{k}\right\}$ is u.d. $(\bmod \Delta)$. Then since

$$
N\left(\frac{1}{2}, \frac{z_{n}+z_{n+1}}{2}\right)-N\left(1 / 2, z_{n}\right)=N\left(\frac{z_{n}+z_{n+1}}{2}\right)-N\left(z_{n}\right),
$$

we have

$$
\begin{aligned}
\frac{1}{2} & \sim \frac{N\left(1 / 2,\left(z_{n}+z_{n+1}\right) / 2\right)}{N\left(\left(z_{n}+z_{n+1}\right) / 2\right)}=\frac{N\left(1 / 2, z_{n}\right)}{N\left(\left(z_{n}+z_{n+1}\right) / 2\right)}+\frac{N\left(\left(z_{n}+z_{n+1}\right) / 2\right)-N\left(z_{n}\right)}{N\left(\left(z_{n}+z_{n+1}\right) / 2\right)} \\
& =\frac{N\left(1 / 2, z_{n}\right)}{N\left(z_{n}\right)} \cdot \frac{N\left(z_{n}\right)}{N\left(\left(z_{n}+z_{n+1}\right) / 2\right)}+1-\frac{N\left(z_{n}\right)}{N\left(\left(z_{n}+z_{n+1}\right) / 2\right)} \\
& =1+\frac{N\left(z_{n}\right)}{N\left(\left(z_{n}+z_{n+1}\right) / 2\right)}\left(\frac{N\left(1 / 2, z_{n}\right)}{N\left(z_{n}\right)}-1\right) \sim 1-\frac{1}{2} \frac{N\left(z_{n}\right)}{N\left(\left(z_{n}+z_{n+1}\right) / 2\right)}
\end{aligned}
$$

as $n \longrightarrow \infty$, and so

$$
N\left(z_{n}\right) \sim N\left(\frac{z_{n}+z_{n+1}}{2}\right)
$$

In the same way it can be shown that

$$
N\left(\frac{z_{n}+z_{n+1}}{2}\right) \sim N\left(z_{n+1}\right),
$$

and consequently $N\left(z_{n}\right) \sim N\left(z_{n+1}\right)$. 
3. The following theorem, due in a slightly different form to Fejér (see [ 1, p. 88-89]), expresses the fact that if $f$ is sufficiently smooth and $[f(x)]$ is constant over increasingly long intervals as $x$ increases, such that the length of the $n$-th interval is of smaller order of magnitude than the total length of all preceding intervals, then $f(k)$ is u.d. $(\bmod 1)$ :

Suppose that $f(x)$ has the following properties:

(i) $f$ is continuously differentiable for $x>x_{0}$,

(ii) $f(x) \uparrow \infty$ as $x \uparrow \propto$,

(iii) $f^{\prime}(x) \searrow 0$ as $x \uparrow \infty$,

(iv) $x f^{\prime}(x) \rightarrow \infty$ as $x \rightarrow \infty$.

Then $f(k)$ is u.d. $(\bmod 1)$.

The following theorem uses the same general idea:

THEOREM 2. Suppose that, for a given subdivision $\Delta$ and a sequence $\left\{x_{k}\right\}$, $N\left(z_{n}\right)-N\left(z_{n-1}\right) \longrightarrow \infty$ as $n \rightarrow \infty$. Then $\left\{x_{k}\right\}$ is u.d. $(\bmod \Delta)$ if the following conditions are satisfied:

(i) $N\left(z_{n-1}\right) \sim N\left(z_{n}\right)$ as $n \rightarrow \infty$,

(ii) except possibly on a sequence of intervals $\left[z_{n_{t}-1}, z_{n_{t}}\right)$ such that

$$
\sum_{t=1}^{m}\left(N\left(z_{n_{t}}\right)-N\left(z_{n_{t}-1}\right)\right)=o\left(N\left(z_{n_{m}}\right)\right)
$$

the relation

$$
\max \left(x_{k}-x_{k-1}\right) \sim \min \left(x_{k}-x_{k-1}\right)
$$

holds as $n \longrightarrow \infty$, the maximum and minimum being taken independently, for given $n \neq n_{1}, n_{2}, \cdots$, over all $k$ for which at least one of $x_{k-1}$ and $x_{k}$ is in $\left[z_{n-1}, z_{n}\right]$.

Give the name $\delta_{n}$ to the interval $\left[z_{n-1}, z_{n}\right]$, and put

$$
\begin{gathered}
N\left(\alpha, \delta_{n}\right)=N\left(z_{n-1}+\alpha\left(z_{n}-z_{n-1}\right)\right)-N\left(z_{n-1}\right), \\
N\left(\delta_{n}\right)=N\left(1, \delta_{n}\right)=N\left(z_{n}\right)-N\left(z_{n-1}\right) .
\end{gathered}
$$

It will be shown that

$$
\lim _{\substack{n \rightarrow \infty \\ n \neq n_{1}, n_{2}, \ldots}} \frac{N\left(\alpha, \delta_{n}\right)}{N\left(\delta_{n}\right)}=\alpha ;
$$


in other words, that in the limit the $x_{k}$ 's which lie in $\delta_{n} \neq \delta_{n_{t}}$ are u.d.there. This implies the theorem, for using it, (1), and (i) we have, for $x \in \delta_{n}$,

$$
\begin{aligned}
\frac{N(\alpha, x)}{N(x)} & =\frac{1}{N(x)}\left\{\sum_{\nu=1}^{n-1} N\left(\alpha, \delta_{n}\right)+N\left(\min \left(x, z_{n-1}+\alpha\left(z_{n}-z_{n-1}\right)\right)\right)-N\left(z_{n-1}\right)\right\} \\
& =\frac{1}{N(x)} \sum^{\circ} N\left(\alpha, \delta_{\nu}\right)+o(1) \\
& =\frac{\sum^{\circ}(\alpha+o(1)) N\left(\delta_{\nu}\right)}{\sum^{\circ} N\left(\delta_{\nu}\right)+o\left(\sum^{\circ} N\left(\delta_{\nu}\right)\right)+N(x)-N\left(z_{n-1}\right)}+o(1) \\
& =\frac{\alpha}{1+o(1)}+o(1)=\alpha+o(1)
\end{aligned}
$$

where $\sum^{\circ}$ denotes summation from $\nu=1$ to $\nu=n-1, \nu \neq n_{1}, n_{2}, \ldots$.

To prove (2), suppose that $n \neq n_{1}, n_{2}, \cdots$, that $z_{n-1} \in\left(x_{k_{n}}, x_{k_{n}+1}\right]$, and that

$$
\min _{k_{n} \leq k \leq k_{n+1}}\left(x_{k}-x_{k-1}\right)=X_{n}
$$

Then for $k_{n} \leq k \leq k_{n+1}$, we have $x_{k}-x_{k-1}=\left(1+\epsilon_{k n}\right) X_{n}$, where $\epsilon_{k n}$ is a positive quantity tending to zero as $n \longrightarrow \infty$. Put

$$
\epsilon_{n}=\max _{k_{n} \leq k \leq k_{n+1}} \epsilon_{k n}
$$

and put $\Delta x_{k}=x_{k}-x_{k-1}$. Now if

$$
x_{k_{n}+t} \leq z_{n-1}+\alpha\left(z_{n}-z_{n-1}\right)<x_{k_{n}+t+1},
$$

then

$$
\begin{aligned}
\alpha\left(z_{n}-z_{n-1}\right) & =\left(x_{k_{n}+1}-z_{n-1}\right)+\sum_{k=k_{n}+2}^{k_{n}+t} \Delta x_{k}+\left(z_{n-1}+\alpha\left(z_{n}-z_{n-1}\right)-x_{k_{n}+t}\right) \\
& =\sum_{s=1}^{t} \Delta x_{k_{n}+s}+\epsilon_{n}^{\prime} X_{n},
\end{aligned}
$$

where $\epsilon_{n}^{\prime}=O(1)$ as $n \rightarrow \infty$. But 


$$
t X_{n} \leq \sum_{s=1}^{t} \Delta x_{k_{n}+s} \leq t X_{n}+t \epsilon_{n} X_{n} \leq t X_{n}+u \epsilon_{n} X_{n}
$$

where $u=N\left(z_{n}\right)-N\left(z_{n-1}\right)$. Hence

$$
\alpha \frac{z_{n}-z_{n-1}}{X_{n}}-\epsilon_{n}^{\prime}-u \epsilon_{n} \leq t \leq \alpha \frac{z_{n}-z_{n-1}}{X_{n}}-\epsilon_{n}^{\prime} .
$$

Similarly,

$$
\frac{z_{n}-z_{n-1}}{X_{n}}-\epsilon_{n}^{\prime}-u \epsilon_{n} \leq u \leq \frac{z_{n}-z_{n-1}}{X_{n}}-\epsilon_{n}^{\prime},
$$

so that

$$
\frac{\alpha\left(z_{n}-z_{n-1}\right) / X_{n}-\epsilon_{n}^{\prime}-u \epsilon_{n}}{\left(z_{n}-z_{n-1}\right) / X_{n}-\epsilon_{n}^{\prime}} \leq \frac{t}{u} \leq \frac{\left(z_{n}-z_{n-1}\right) / X_{n}-\epsilon_{n}^{\prime}}{\left(z_{n}-z_{n-1}\right) / X_{n}-\epsilon_{n}^{\prime}-u \epsilon_{n}} .
$$

Since $N\left(z_{n}\right)-N\left(z_{n-1}\right) \rightarrow \infty$ as $n \rightarrow \infty$, also $\left(z_{n}-z_{n-1}\right) / X_{n} \rightarrow \infty$, and so

$$
\frac{\alpha+o(1)-u \epsilon_{n} X_{n} /\left(z_{n}-z_{n-1}\right)}{1+o(1)} \leq \frac{t}{u} \leq \frac{\alpha+o(1)}{1+o(1)-u \epsilon_{n} X_{n} /\left(z_{n}-z_{n-1}\right)} .
$$

But since

$$
u X_{n} \leq \sum_{k=k_{n}+1}^{k_{n+1}} \Delta x_{k} \leq z_{n}-z_{n-1}
$$

$u X_{n}=O\left(z_{n}-z_{n-1}\right) ;$ thus

$$
\frac{\alpha+o(1)}{1+o(1)} \leq \frac{t}{u} \leq \frac{\alpha+o(1)}{1+o(1)}
$$

and therefore

$$
\frac{N\left(\alpha, \delta_{n}\right)}{N\left(\delta_{n}\right)}=\frac{t}{u} \sim \alpha .
$$

This completes the proof.

In case $\Delta=\Delta_{0}$ and $x_{k}=f(k)$, it is easily seen that the hypotheses of Fejér's theorem imply two of the hypotheses of Theorem 2, namely that $N\left(z_{n}\right)-$ 
$N\left(z_{n-1}\right) \uparrow \infty$ and $N\left(z_{n-1}\right) \sim N\left(z_{n}\right)$ as $n \rightarrow \infty$. But I do not know whether Theorem 2 includes Fejér's theorem; the most that $I$ can show is that the exceptional sequence $\left\{z_{n_{t}}\right\}=\left\{n_{t}\right\}$ mentioned in (ii) of Theorem $\mathrm{o}_{\mathrm{o}} 2$ is in this case of density zero, which does not imply ( 1 ) for all functions $f$ satisfying the hypotheses of Fejer's theorem. Certainly, however, Theorem 2 deals with cases not covered by the following direct extension of Fejér's theorem, since it does not require the monotonicity of either $z_{n}-z_{n-1}$ or $\Delta x_{k}$.

THEOREM 3. The sequence $\left\{x_{k}\right\}$ is u.d. $(\bmod \Delta)$ if the following conditions are satisfied:

(i) $z_{n}-z_{n-1} \geq z_{n-1}-z_{n-2}$ for $n=2,3, \cdots$,

(ii) $\Delta x_{k} \downarrow 0$ as $k \uparrow \infty$,

(iii) $N\left(z_{n-1}\right) \sim N\left(z_{n}\right)$ as $n \rightarrow \infty$.

We sketch the proof. Let $\psi$ be the continuous polygonal function such that $\psi\left(x_{k}\right)=k$; then $0 \leq \psi(x)-N(x)<1$. Let $\left\{\epsilon_{k}\right\}$ be such that $\epsilon_{k}=o\left(\Delta x_{k}\right)$ and $0<\epsilon_{k}<\Delta x_{k} / 2$ for $k=1,2, \cdots$. Define $\psi_{1}$ as follows:

$$
\begin{array}{r}
\psi_{1}(x)=\frac{1}{2 \epsilon_{k}} \int_{x-\epsilon_{k}}^{x+\epsilon_{k}} \psi(t) d t \text { for } x \in\left[x-\frac{1}{2} \Delta x_{k}, x_{k}+\frac{1}{2} \Delta x_{k+1}\right] \\
(k=2,3, \cdots) .
\end{array}
$$

Then $\psi_{1}$ is continuously differentiable, and is identical with $\psi$ except at the corners of $\psi$, where it is smooth. For $0 \leq \alpha \leq 1, n=1,2,3, \cdots$, put

$$
\rho(n+\alpha)=\psi_{1}\left(z_{n-1}+\alpha\left(z_{n}-z_{n-1}\right)\right)
$$

$\rho$ is continuously differentiable except at $x=1,2, \ldots$ A function $\rho_{1}$ can now be defined in terms of $\rho$, just as $\psi_{1}$ was determined from $\psi$, so that $\rho_{1}$ is everywhere continuously differentiable, and $\rho_{1}$ differs from $\rho$ only on an interval about $x=$ $n(n=1,2, \cdots)$ whose length $\epsilon_{n}^{\prime}$ is of lower order of magnitude than $\Delta x_{k_{n}}$ if $z_{n} \in\left[x_{k_{n}-1}, x_{k_{n}}\right)$. If $x=n+\alpha$ is such that

$$
\rho_{1}(x)=\rho(x), \quad \psi_{1}\left(z_{n-1}+\alpha\left(z_{n}-z_{n-1}\right)\right)=\psi\left(z_{n-1}+\alpha\left(z_{n}-z_{n-1}\right)\right),
$$

and

$$
z_{n-1}+\alpha\left(z_{n}-z_{n-1}\right) \in\left(x_{k-1}, x_{k}\right)
$$

then 


$$
\rho_{1}^{\prime}(x)=\frac{z_{n}-z_{n-1}}{\Delta x_{k}}
$$

it follows that $\rho_{1}^{\prime}(x) \nearrow \infty$. Moreover, since

$$
\frac{\rho_{1}(n+1)}{\rho_{1}(n)} \sim \frac{\psi\left(z_{n}\right)}{\psi\left(z_{n-1}\right)} \sim \frac{N\left(z_{n}\right)}{N\left(z_{n-1}\right)} \rightarrow 1,
$$

it follows that $\rho_{1}^{\prime}(x) / \rho_{1}(x) \rightarrow 0$ as $x \rightarrow \infty$. But if $f$ is the function inverse to $\rho_{1}$, these facts imply that $f(x) \uparrow \infty, f^{\prime}(x) \searrow 0$, and $x f^{\prime}(x) \rightarrow \infty$ as $x \uparrow \infty$. Since $f(k) \longrightarrow x_{k}$ as the arbitrary numbers $\epsilon_{k}$ and $\epsilon_{n}^{\prime}$ approach zero, the conclusion follows from Fejér's theorem.

A trivial variation of Theorem 3 has, instead of (i) and (ii), the hypotheses

(i') $z_{n}-z_{n-1} \uparrow \propto$,

(ii') $\Delta x_{k-1} \geq \Delta x_{k}$ for $k=2,3, \cdots$.

For then it will still be true that $\rho_{1}^{\prime}(x) \nearrow \infty$ as $x \uparrow \propto$.

4. It follows from Theorem 2 (and also from the variation of Theorem 3 just mentioned) that if $\left.z_{n}-z_{n-1}\right\rceil \infty$ in such a way that $z_{n-1} \sim z_{n}$, the sequence $\{k \theta\}$ is u.d. $(\bmod \Delta)$ for each $\theta>0$. In this section we examine the distribution of $\{k \theta\}(\bmod \Delta)$ when $\delta(x) \downarrow 0$. This is a problem of a very different kind from the earlier one; the result is expressed in the following metric theorem:

THEOREM 4. If $\delta(x) \searrow 0$ and $\delta(x)=O\left(x^{-1}\right)$ then $\{k \theta\}$ is u.d. $(\bmod \Delta)$ for almost all $\theta>0$.

The proof depends on a principle used in an earlier paper [2]:

If $C$ and $\epsilon$ are positive constants and $\left\{f_{k}\right\}$ is a sequence of real-valued functions such that

$$
\left|\int_{a}^{b} e^{i\left(f_{j}(x)-f_{k}(x)\right)} d x\right| \leq \frac{C}{\max \left(1,|j-k|^{\epsilon}\right)}, \quad(j, k=1,2, \cdots)
$$

then $\left\{f_{k}(x)\right\}$ is u.d. $(\bmod 1)$ for almost all $x \in(a, b)$.

This will be applied with $f_{k}(x)=\phi(k x)$, where $\phi$ is the function defined in $\S 1$; it was noted there that the u.d. $(\bmod \Delta)$ of $\left\{x_{k}\right\}$ is equivalent to the u.d. $(\bmod 1)$ of $\left\{\phi\left(x_{k}\right)\right\}$. Let $a$ and $b$ be arbitrary positive numbers with $a<b$, and put 


$$
J_{j k}=\int_{a}^{b} e^{i\left(f_{j}(x)-f_{k}(x)\right)} d x
$$

since $J_{k j}$ and $J_{j k}$ are complex conjugates, it suffices to consider the case $j>k$. For fixed $j$ and $k$, denote by $\xi_{0}, \cdots \xi_{r}$ all the numbers of the form $z_{m} / j$ or $z_{m} / k$ in the interval $(a, b)$, so named that $\xi_{0}<\cdots<\xi_{r}$. Then the function

$$
f_{j}(x)-f_{k}(x)=\left(\frac{j}{\delta(j x)}-\frac{k}{\delta(k x)}\right) x-\left(\frac{[j x]_{\Delta}}{\delta(j x)}-\frac{[k x]_{\Delta}}{\delta(k x)}\right)=x A(x)+B(x)
$$

is linear in each interval $\left[\xi_{l-1}, \xi_{l}\right), A(x)$ and $B(x)$ being certain constants $A_{l}$ and $B_{l}$ there. Hence

$$
J_{j k}=\sum_{l=1}^{r} \int_{\xi_{l-1}}^{\xi_{l}} e^{i\left(A_{l} x+B_{l}\right)} d x=\sum_{l=1}^{r} \frac{e^{i\left(A_{l} \xi_{l}+B_{l}\right)}-e^{i\left(A_{l} \xi_{l-1}+B_{l}\right)}}{i A_{l}}
$$

Since $f$ is continuous,

$$
A_{l} \xi_{l}+B_{l}=A_{l+1} \xi_{l}+B_{l+1},
$$

and so for $1 \leq t \leq r$,

$$
\sum_{l=1}^{t}\left[e^{i\left(A_{l} \xi_{l}+B_{l}\right)}-e^{i\left(A_{l} \xi_{l-1}+B_{l}\right)}\right]=e^{i\left(A_{t} \xi_{t}+B_{t}\right)}-e^{i\left(A_{1} \xi_{0}+B_{1}\right)}
$$

Thus, using the relation

$$
\sum_{m=1}^{n} a_{m} b_{m}=\sum_{m=1}^{n-1}\left(\sum_{\mu=1}^{m} a_{\mu}\right)\left(b_{m}-b_{m+1}\right)+b_{n} \sum_{\mu=1}^{n} a_{\mu},
$$

we have

$$
\begin{aligned}
J_{j k}=\frac{1}{i} \sum_{t=1}^{r-1}\left(e^{i\left(A_{t} \xi_{t}+B_{t}\right)}-e^{i\left(A_{1} \xi_{0}+B_{1}\right)}\right)\left(\frac{1}{A_{t}}-\frac{1}{A_{t+1}}\right) \\
+\left(e^{i\left(A_{r} \xi_{r}+B_{r}\right)}-e^{i\left(A_{1} \xi_{0}+B_{1}\right)}\right) \frac{1}{i A_{r}},
\end{aligned}
$$

and so 


$$
\left|J_{j k}\right| \leq 2 \sum_{t=1}^{r-1}\left|\frac{1}{A_{t}}-\frac{1}{A_{t+1}}\right|+\frac{2}{\left|A_{r}\right|} .
$$

By the facts that $\xi_{t} \geq a>0, \delta(x) \searrow 0$ as $x \rightarrow \infty$, and

$$
A_{t}=\frac{j}{\delta\left(j \xi_{t-1}\right)}-\frac{k}{\delta\left(k \xi_{t-1}\right)}
$$

it is clear that

$$
A_{t}>C(j-k)>0
$$

for $t=1,2, \cdots, r$, so that (3) will follow from (4) if it can be shown that for some $c, \epsilon>0$, the inequality

$$
\sum_{t=1}^{r-1}\left|\frac{1}{A_{t}}-\frac{1}{A_{t+1}}\right|<\frac{c}{(j-k)^{\epsilon}}
$$

holds. Moreover, writing

$$
C_{t}=\frac{1}{A_{t}}-\frac{1}{A_{t+1}}
$$

and

$$
\sum_{t=1}^{r-1}\left|C_{t}\right|=\sum_{t=1}^{r} C_{t}-2 \sum^{\prime} C_{t}=\frac{1}{A_{1}}-\frac{1}{A_{r}}-2 \sum^{\prime} C_{t}
$$

where $\Sigma^{\prime}$ is the sum over those $t$ for which $C_{t}<0$, we see that it suffices to show that

$$
\Sigma^{\prime}\left|C_{t}\right|<\frac{c}{(j-k)^{\epsilon}} .
$$

We consider three cases. Suppose first that $t$ is such that $\xi_{t+1}=z_{m} / j$ for some $m$, but that for no $l$ is $\xi_{t+1}=z_{l} / k$. Then

$$
A_{t}=\frac{j}{\delta\left(z_{m-1}\right)}-\frac{k}{\delta\left(k \xi_{t}\right)}, A_{t+1}=\frac{j}{\delta\left(z_{m}\right)}-\frac{k}{\delta\left(k \xi_{t}\right)},
$$

so that $A_{t+1} \geq A_{t}$, and the term $C_{t}$ does not occur in $\Sigma^{\prime}$. If 


$$
\xi_{t+1}=z_{m} / j=z_{l} / k
$$

then $z_{m}>z_{l}$ and

$$
\begin{aligned}
C_{t} & =\frac{1}{j / \delta\left(z_{m-1}\right)-k / \delta\left(z_{l-1}\right)}-\frac{1}{j / \delta\left(z_{m}\right)-k / \delta\left(z_{l}\right)} \\
& \geq \frac{-k\left(1 / \delta\left(z_{l}\right)-1 / \delta\left(z_{l-1}\right)\right)}{\left(j / \delta\left(z_{m-1}\right)-k / \delta\left(z_{l-1}\right)\right)\left(j / \delta\left(z_{m}\right)-k / \delta\left(z_{l}\right)\right)} .
\end{aligned}
$$

Finally, if $\xi_{t+1}=z_{l} / k$ for some $l$, but $\xi_{t+1} \neq z_{m} / j$ for every $m$, then

$$
C_{t}=\frac{-k\left(1 / \delta\left(z_{l}\right)-1 / \delta\left(z_{l-1}\right)\right)}{\left(j / \delta\left(j \xi_{t+1}\right)-k / \delta\left(z_{l-1}\right)\right)\left(j / \delta\left(j \xi_{t+1}\right)-k / \delta\left(z_{l}\right)\right)} .
$$

Thus, writing $\delta\left(x^{+}\right)$and $\delta\left(x^{-}\right)$for $\lim _{\xi \rightarrow x^{+}} \delta(\xi)$ and $\lim _{\xi \rightarrow x^{-}} \delta(\xi)$, we have

$$
\begin{aligned}
\sum^{\prime}\left|C_{t}\right| \leq k \sum^{\prime \prime} & \frac{1 / \delta\left(z_{l}\right)-1 / \delta\left(z_{l-1}\right)}{\left(j / \delta\left(j \xi_{t+1}^{-}\right)-k / \delta\left(z_{l-1}\right)\right)\left(j / \delta\left(j \xi_{t+1}^{+}\right)-k / \delta\left(z_{l}\right)\right)} \\
& =\sum^{\prime \prime} \frac{1 / \delta\left(z_{l}\right)-1 / \delta\left(z_{l-1}\right)}{\left(j / \delta\left(j z_{l}^{-} / k\right)-k / \delta\left(z_{l-1}\right)\right)\left(j / \delta\left(j z_{l}^{+} / k\right)-k / \delta\left(z_{l}\right)\right)},
\end{aligned}
$$

where $\sum$ " denotes summation with respect to $l$ with $z_{l} / k \in(a, b)$. But

$$
\delta\left(j z_{l}^{-} / k\right) \leq \delta\left(z_{l-1}\right)
$$

and

$$
\delta\left(j z_{l}^{+} / k\right) \leq \delta\left(z_{l}\right)
$$

and so

$$
\begin{aligned}
\sum^{\prime}\left|C_{t}\right| & \leq k \sum^{\prime \prime} \frac{1 / \delta\left(z_{l}\right)-1 / \delta\left(z_{l-1}\right)}{(j-k)^{2} / \delta\left(z_{l-1}\right) \delta\left(z_{l}\right)} \\
& =\frac{k}{(j-k)^{2}} \sum^{\prime \prime}\left\{\delta\left(z_{l-1}\right)-\delta\left(z_{l}\right)\right\} \leq \frac{2 k \delta(k a)}{(j-k)^{2}} .
\end{aligned}
$$

If now $\delta(x)=O(1 / x)$, then

$$
\sum^{\prime}\left|C_{t}\right|=O\left(\frac{1}{(j-k)^{2}}\right)
$$


and the proof is complete.

5. The preceding result can be generalized considerably by using the following transfer theorem:

THEOREM 5. Suppose that $\left\{x_{k}\right\}$ is u.d. $(\bmod \Delta)$, where $\Delta=\left\{z_{n}\right\}$, and that $f$ is a function which is differentiable except possibly at the points $z_{1}, z_{2}, \ldots$, such that $f(x) \uparrow \infty$ as $x \uparrow \infty$ and

$$
\inf _{x \in\left(z_{n-1}, z_{n}\right)} f^{\prime}(x) \sim \sup _{x \in\left(z_{n-1}, z_{n}\right)} f^{\prime}(x) .
$$

Then the sequence $\left\{x_{k}^{*}\right\}=\left\{f\left(x_{k}\right)\right\}$ is u.d. $\left(\bmod \Delta^{*}\right)$, where $\Delta^{*}=\left\{f\left(z_{n}\right)\right\}$.

Put

$N(\alpha, x)=\sum 1, N(1, x)=N(x), N^{*}(\alpha, x)=\sum^{*} 1, N^{*}(1, x)=N^{*}(x)$,

where $\Sigma$ denotes summation with $x_{k} \leq x$ and $\left\langle x_{k}\right\rangle_{\Delta}<\alpha$ and $\Sigma^{*}$ denotes summation with $x_{k}^{*} \leq x,\left\langle x_{k}^{*}\right\rangle_{\Delta^{*}}<\alpha$. Since $f$ is an increasing function,

$$
N^{*}(f(x))=\sum_{f\left(x_{k}\right) \leq f(x)} 1=\sum_{x_{k} \leq x} 1=N(x) .
$$

By assumption, the relation

$$
\lim _{x \rightarrow \infty} \frac{N(\alpha, x)}{N(x)}=\alpha
$$

holds for $\alpha \in[0,1]$. So we need only show that $N^{*}(\alpha, f(x)) \sim N(\alpha, x)$ as $x \rightarrow \infty$, and by Theorem 1 it suffices to prove this as $x$ runs through the sequence $\left\{z_{n}\right\}$. But

$$
N\left(\alpha, z_{n}\right)=\sum_{m=1}^{n}\left\{N\left(z_{m-1}+\alpha\left(z_{m}-z_{m-1}\right)\right)-N\left(z_{m-1}\right)\right\},
$$

and so

$$
\begin{aligned}
& N^{*}\left(\alpha, f\left(z_{n}\right)\right)=\sum_{m=1}^{n}\left\{N^{*}\left(z_{m-1}^{*}+\alpha\left(z_{m}^{*}-z_{m-1}^{*}\right)\right)-N^{*}\left(z_{m-1}^{*}\right)\right\} \\
& =N\left(\alpha, z_{n}\right)+\sum_{m=1}^{n}\left\{N^{*}\left(z_{m-1}^{*}+\alpha\left(z_{m}^{*}-z_{m-1}^{*}\right)\right)-N\left(z_{m-1}+\alpha\left(z_{m}-z_{m-1}\right)\right)\right\} .
\end{aligned}
$$


Thus the problem reduces to showing that

$$
\sum_{m=1}^{n}\left\{N^{*}\left(z_{m-1}^{*}+\alpha\left(z_{m}^{*}-z_{m-1}^{*}\right)\right)-N\left(z_{m-1}+\alpha\left(z_{m}-z_{m-1}\right)\right)\right\}=o\left(N\left(\alpha, z_{n}\right)\right)
$$

or what is the same thing, that

(6) $\sum_{m=1}^{n}\left\{N\left(f^{-1}\left(z_{m-1}^{*}+\alpha\left(z_{m}^{*}-z_{m-1}^{*}\right)\right)\right)-N\left(z_{m-1}+\alpha\left(z_{m}-z_{m-1}\right)\right)\right\}=o\left(N\left(z_{n}\right)\right)$. Put

$$
\begin{aligned}
f^{-1}\left(z_{m-1}^{*}+\alpha\left(z_{m}^{*}-z_{m-1}^{*}\right)\right) & =u_{m}(\alpha), \\
z_{m-1}+\alpha\left(z_{m}-z_{m-1}\right) & =v_{m}(\alpha) .
\end{aligned}
$$

If it can be shown that

$$
\left|u_{m}(\alpha)-v_{m}(\alpha)\right|<\epsilon_{m}\left(z_{m}-z_{m-1}\right),
$$

where $\epsilon_{m} \longrightarrow 0$ as $m \longrightarrow \infty$, then for every $\epsilon>0$,

$$
\begin{aligned}
\sum_{m=1}^{n}\left\{N\left(u_{m}(\alpha)\right)-N\left(v_{m}(\alpha)\right)\right\} \\
\quad=O\left(\sum_{m=1}^{n}\left\{N\left(v_{m}(\alpha)+\epsilon\left(z_{m}-z_{m-1}\right)\right)-N\left(v_{m}(\alpha)\right)\right\}\right) \\
=O\left(N\left(\epsilon, z_{n}\right)\right)=O\left(\epsilon N\left(z_{n}\right)\right),
\end{aligned}
$$

which implies (6).

Now

$$
u_{m}(0)=v_{m}(0), u_{m}(1)=v_{m}(1)
$$

and

$$
\begin{aligned}
u_{m}(\alpha)-v_{m}(\alpha)=f^{-1}\left(f\left(z_{m-1}\right)\right. & \left.+\alpha\left(f\left(z_{m}\right)-f\left(z_{m-1}\right)\right)\right) \\
& -\left(z_{m-1}+\alpha\left(z_{m}-z_{m-1}\right)\right)
\end{aligned}
$$

hence 


$$
u_{m}^{\prime}(\alpha)-v_{m}^{\prime}(\alpha)=\frac{f\left(z_{m}\right)-f\left(z_{m-1}\right)}{f^{\prime}\left\{f^{-1}\left(f\left(z_{m-1}\right)+\alpha\left(f\left(z_{m}\right)-f\left(z_{m-1}\right)\right)\right)\right\}}-\left(z_{m}-z_{m-1}\right) .
$$

To maximize $u_{m}(\alpha)-v_{m}(\alpha)$, we must have

$$
f\left(z_{m}\right)-f\left(z_{m-1}\right)-\left(z_{m}-z_{m-1}\right) f^{\prime}\left\{f^{-1}\left(f\left(z_{m-1}\right)+a\left(f\left(z_{m}\right)-f\left(z_{m-1}\right)\right)\right)\right\}=0 \text {. }
$$

There is a $Z_{0} \in\left(z_{m-1}, z_{m}\right)$ such that

$$
\frac{f\left(z_{m}\right)-f\left(z_{m-1}\right)}{z_{m}-z_{m-1}}=f^{\prime}\left(Z_{0}\right)
$$

and a corresponding $\alpha_{0} \in(0,1)$ such that

$$
f\left(z_{m-1}\right)+\alpha_{0}\left(f\left(z_{m}\right)-f\left(z_{m-1}\right)\right)=f\left(Z_{0}\right),
$$

( so that $u_{m}^{\prime}\left(\alpha_{0}\right)-v_{m}^{\prime}\left(\alpha_{0}\right)=0$ ) for which

$$
\left|u_{m}(\alpha)-v_{m}(\alpha)\right| \leq\left|u_{m}\left(\alpha_{0}\right)-v_{m}\left(\alpha_{0}\right)\right|=\left|Z_{0}-v_{m}\left(\alpha_{0}\right)\right|
$$

for all $\alpha \in(0,1)$. But

$$
\begin{aligned}
v_{m}\left(\alpha_{0}\right) & =z_{m-1}+\frac{f\left(Z_{0}\right)-f\left(z_{m-1}\right)}{f\left(z_{m}\right)-f\left(z_{m-1}\right)}\left(z_{m}-z_{m-1}\right) \\
& =z_{m-1}+\frac{f\left(Z_{0}\right)-f\left(z_{m-1}\right)}{f^{\prime}\left(Z_{0}\right)},
\end{aligned}
$$

so that

$$
Z_{0}-v_{m}\left(\alpha_{0}\right)=Z_{0}-z_{m-1}-\frac{f\left(Z_{0}\right)-f\left(z_{m-1}\right)}{f^{\prime}\left(Z_{0}\right)}
$$

and

$$
\left|u_{m}(\alpha)-v_{m}(\alpha)\right| \leq \sup _{Z \in \delta_{m}}\left(\left|Z-z_{m-1}\right|\left|1-\frac{f(Z)-f\left(z_{m-1}\right)}{\left(Z-z_{m-1}\right) f^{\prime}(Z)}\right|\right),
$$

whence

$$
\left|\frac{u_{m}(\alpha)-v_{m}(\alpha)}{z_{m}-z_{m-1}}\right| \leq \sup _{\substack{Z \in \delta_{m} \\ W \in \delta_{m}}}\left|1-\frac{f^{\prime}(W)}{f^{\prime}(Z)}\right|
$$


and this last upper bound is $o(1)$ as $m \longrightarrow \infty$. Thus $(7)$ holds, and the proof is complete.

If the $f$ of Theorem 5 is taken to be an arbitrary increasing polygonal function, with vertices on the abscissas $x=z_{1}, z_{2}, \cdots$, then the condition ( 5 ) on the derivative is trivially satisfied. Such a transformation merely represents a change of scale inside each interval $\delta_{n}$, and the distribution modulo $\Delta$ of any sequence $\left\{x_{k}\right\}$ is identical with the distribution of $\left\{f\left(x_{k}\right)\right\}$ modulo $\Delta^{*}$.

In case $f^{\prime}$ is monotone, $(5)$ can be replaced by the simpler condition

$$
f^{\prime}\left(z_{n-1}\right) \sim f^{\prime}\left(z_{n}\right) \quad \text { as } n \rightarrow \infty
$$

Combining this version of Theorem 5 with Theorem 4, we have:

THEOREM 6. The sequence $\{f(k \theta)\}$ is u.d. $(\bmod \Delta)$ for almost all $\theta>0$ if $f(x) \uparrow \infty, f^{\prime}$ is monotonic, and

$$
\begin{gathered}
f^{-1}\left(z_{n}\right)-f^{-1}\left(z_{n-1}\right) \searrow 0, \\
f^{-1}\left(z_{n}\right)-f^{-1}\left(z_{n-1}\right)=O\left(\frac{1}{f^{-1}\left(z_{n}\right)}\right), \\
f^{\prime}\left(f^{-1}\left(z_{n}\right)\right) \sim f^{\prime}\left(f^{-1}\left(z_{n-1}\right)\right),
\end{gathered}
$$

where $f^{-1}$ is the function inverse to $f$.

Corollary. The sequence $\left\{\alpha^{k}\right\}$ is u.d. $(\bmod \Delta)$ for almost all $\alpha>1$ if $z_{n}=g(n)$, where $g$ is an increasing function with monotonic logarithmic derivative such that

$$
\frac{g^{\prime}(x)}{g(x)}=O\left(x^{-1 / 2}\right) .
$$

For writing $\alpha^{k}$ as $e^{k \log \alpha}$, we see that we can take the $f$ of Theorem 6 to be the exponential function, and the conditions displayed there become

$$
\begin{gathered}
\log z_{n}-\log z_{n-1} \searrow 0 \\
\log z_{n}-\log z_{n-1}=O\left(\frac{1}{\log z_{n}}\right), \\
z_{n} \sim z_{n-1}
\end{gathered}
$$

Of these, the third is implied by the first. Since 


$$
\frac{d}{d x} \log g(x) \searrow 0,
$$

it is clear that $\log g(n)-\log g(n-1) \searrow 0$. From the extended law of the mean,

$$
\frac{G(x)-G(x-1)}{H(x)-H(x-1)}=\frac{G^{\prime}(X)}{H^{\prime}(X)}, \quad X \in(x-1, x),
$$

it follows that if $G^{\prime}(x)=O\left(H^{\prime}(x)\right)$, then

$$
G(x)-G(x-1)=O(H(x)-H(x-1)) .
$$

Taking

$$
G(x)=\log g(x), \quad H(x)=\log e^{\sqrt{x}}=\sqrt{x},
$$

we have by ( 8 ) that

$$
\log g(n)-\log g(n-1)=O\left(n^{-1 / 2}\right) .
$$

But it also follows from the relation $G^{\prime}(x)=O\left(H^{\prime}(x)\right)$ that $G(x)=O(H(x))$; hence

$$
\log g(x)=O\left(x^{1 / 2}\right), \quad n^{-1 / 2}=O\left(\left(\log g(n)^{-1}\right),\right.
$$

and the proof is complete.

For sufficiently smooth $g,(8)$ can be replaced by the condition $g(x)=$ $O(\exp \sqrt{x})$.

\section{REFERENCES}

1. J. F. Koksma, Diophantische Approximationen, Ergebnisse der Mathematik, vol.4, no. 4, 1936.

2. W. J. LeVeque, Note on a theorem of Koksma, Proc. Amer. Math. Soc. 1(1950), 380383.

UNIVERSITY OF MichIGAN 
